Document downloaded from:

http://hdl.handle.net/10251/60297

This paper must be cited as:

Casabán Bartual, MC.; Company Rossi, R.; Jódar Sánchez, LA. (2014). Closed form numerical solutions of variable coefficient linear second-order elliptic problems. Applied Mathematics and Computation. 238:266-280. doi:10.1016/j.amc.2014.04.025.

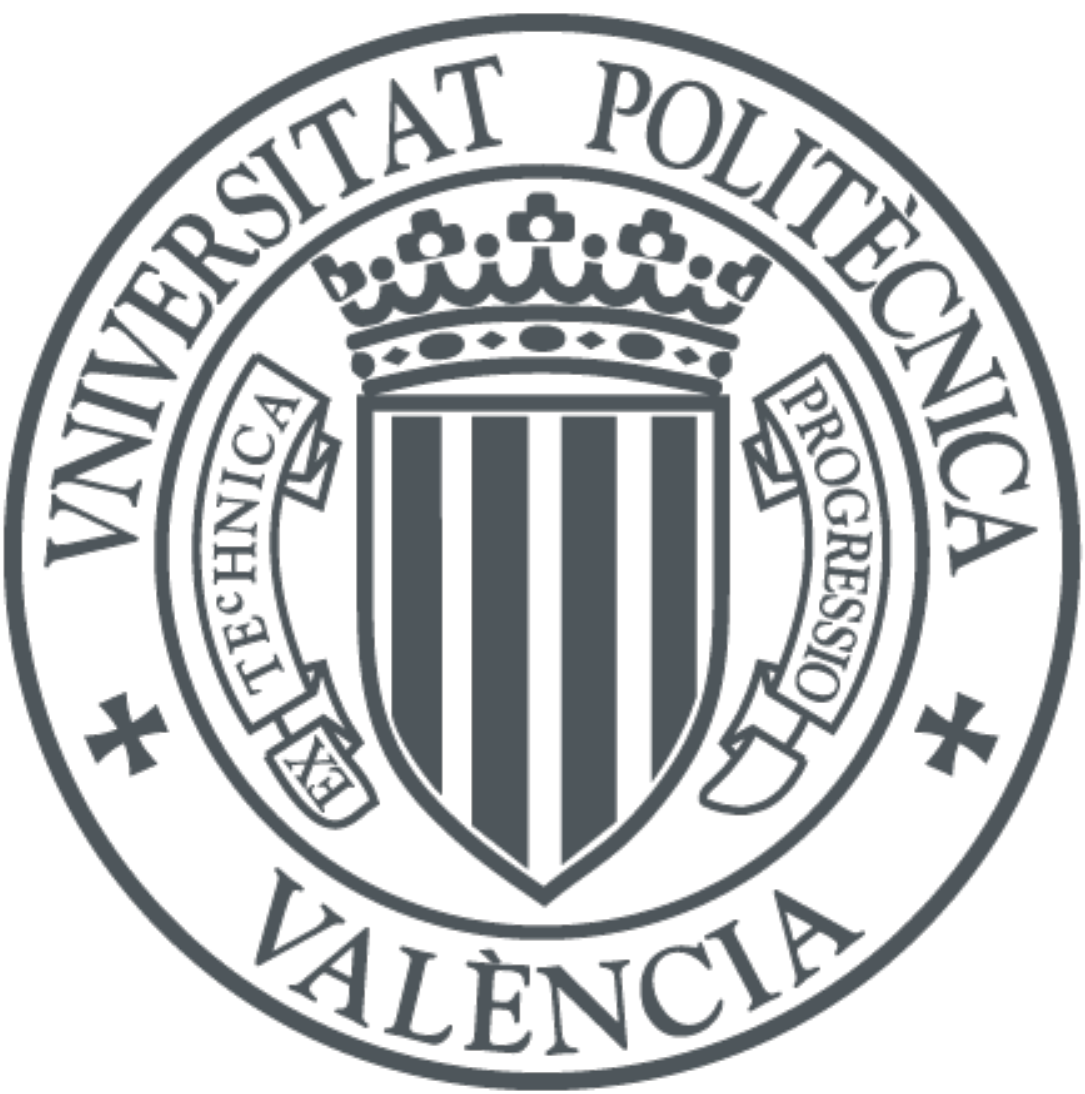

The final publication is available at

http://dx.doi.org/10.1016/j.amc.2014.04.025

Copyright Elsevier

Additional Information 


\title{
Closed form numerical solutions of variable coefficient linear second-order elliptic problems
}

\author{
M.-C. Casabán, R. COMPany, L. Jódar \\ Instituto Universitario de Matemática Multidisciplinar \\ Universitat Politècnica de València \\ Camino de Vera s/n, 46022 Valencia, Spain \\ rcompany@imm.upv.es (R. Company); ljodar@imm.upv.es (L. Jódar)
}

\begin{abstract}
In this work we develop an alternative numerical technique which allows to construct a numerical solution in closed form of variable coefficient linear second-order elliptic problems with Dirichlet boundary conditions. The elliptic partial differential equation is approximated by a consistent explicit difference scheme and using a discrete separation of the variables method we determine a closed form solution of the two resulting discrete boundary value problems with the separated variables, avoiding to have to solve large algebraic systems. One of these boundary value problems is a discrete SturmLiouville problem which guarantees the qualitative properties of the exact solution of elliptic problem. A constructive procedure for the computation of the numerical solution is given and an illustrative example is included.
\end{abstract}

Keywords. Variable coefficient linear elliptic problems, Closed form numerical solutions, Explicit difference scheme, Discrete variable separated method, Discrete Sturm-Liouville problems, Consistency.

\section{Introduction}

Elliptic partial differential equations arise usually from equilibrium or steady-state fluid flow and heat problems and their solutions, in relation to the calculus of variations, frequently maximize or minimize an integral representing the energy of the system. Exact solutions only exits for a few special cases with simple geometries and boundary conditions, or for simplified constants coefficients equations, in which some of the more complicated physical phenomena are neglected. Fortunately, numerical analysis in these equations can offer reliable solutions.

Apart from some techniques such as meshless methods [1, 2] and those based on particular transformations used to solve special problems $[3,4]$, the most used are related mesh methods as the finite difference method $[5,6,7]$, the finite-volume method [8,9] and the finite element method [10, 11].

In this paper we consider an explicit finite difference scheme for the following linear second-order homogeneous elliptic problem with Dirichlet boundary conditions

$$
\begin{aligned}
r(x) u_{y y}(x, y)+\left[p(x) u_{x}(x, y)\right]_{x}-q(x) u(x, y)=0, & a<x<b, c<y<d, \\
u(a, y)=f_{1}(y), & y \in[c, d], \\
u(b, y)=g_{1}(y), & y \in[c, d], \\
u(x, c)=f_{2}(x), & x \in[a, b], \\
u(x, d)=g_{2}(x), & x \in[a, b],
\end{aligned}
$$

${ }^{*}$ Corresponding author. Phone: +34 963879144. E-mail address: macabar@imm.upv.es 
where $r(x), p(x), q(x), f_{1}(y), f_{2}(x), g_{1}(y), g_{2}(x)$ are continuous real functions and

$$
\left.\begin{array}{r}
r(x)>0, p(x)>0, q(x)>0, \quad x \in[a, b] \\
p(x) \text { is differentiable }
\end{array}\right\} .
$$

Discretization of the partial differential equation (pde) together with the boundary conditions give rise to an algebraic discretized problem where the unknowns are the numerical values of the solution at the mesh points. This algebraic problem can be compactly written as a linear system $A u=b$, where the entries of the matrix $A$ and the vector $b$ involve the structure of the pde and the boundary value conditions. A detailed study of this algebraic treatment may be found in [12, Chapter 5], [13, Chapter 5].

An alternative approach to solve the discretized problem as a mere algebraic system, that at the same time tries to preserve the properties of the continuous eigenfunction method for the continuous problem [14], is based on the construction of a discrete separation of the variables method for the resulting discretized problem. This method has been successfully used in [15]-[17] for solving parabolic and hyperbolic problems, and it is considered here for the solution of the elliptic problem (1)-(5) providing a closed form numerical solution.

For the study of the elliptic problem (1)-(5) we decompose the boundary conditions (2)-(5) in the four following cases:

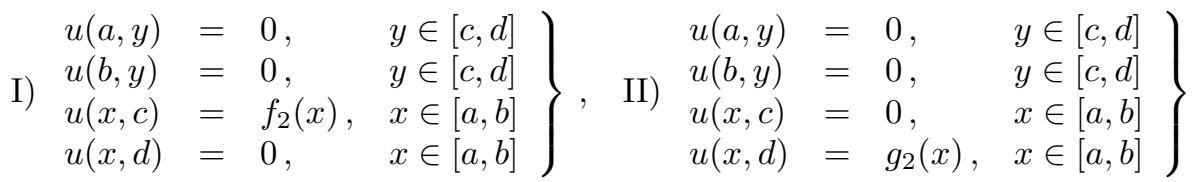

$$
\begin{aligned}
& \text { III) } \left.\left.\begin{array}{lll}
u(a, y) & =f_{1}(y), & y \in[c, d] \\
u(b, y) & =0, & y \in[c, d] \\
u(x, c) & =0, & x \in[a, b] \\
u(x, d) & =0, & x \in[a, b]
\end{array}\right\}, \text { IV) } \begin{array}{ll}
u(a, y)=0, \\
u(x, y)=g_{1}(y), & y \in[c, d] \\
u(x, d)=0, & x \in[a, b] \\
& =0,
\end{array}\right\}
\end{aligned}
$$

Potential advantages of the proposed method are that being explicit and based on a discrete eigenfunction method, the properties of the exact theoretical solution are preserved by the numerical approximation allowing that with just a few nodes the approximation is very good together with a low computational cost.

This paper is organized as follow. Section 2 deals with the discretization of Eq. (1) and the study of the consistency of the constructed numerical scheme. In section 3 we construct numerical solutions considering cases I and II by means of a discrete separation of variables method. The similarity of the first two homogeneous boundary conditions allows to use the same discrete Sturm-Liouville problem in both cases whose eigenfunctions are determined throughout the eigenpairs of an algebraic eigenvalue problem. Cases III and IV are treated in section 4 because both problems have the same last two homogeneous boundary conditions and the underlying discrete Sturm-Liouville problem. In these cases the eigenfunctions are analytically determined. Section 5 includes a constructive numerical algorithm of the original problem. We also include an illustrative numerical example. Section 6 summarizes the main conclusions of the paper.

\section{Discretization and consistency}

Let us begin this section by subdividing the plane domain $[a, b] \times[c, d]$ into a rectangular mesh of equal rectangles of sides $\Delta x=h, \Delta y=k$. Let $N$ and $M$ be natural numbers and let $\alpha$ be positive real number such that

$$
h=\frac{b-a}{N+1}, \quad k=\frac{d-c}{M+1}, \quad \alpha=\frac{k}{h} .
$$


Then a typical mesh point $\left(x_{i}, y_{j}\right)$ verifies

$$
\begin{array}{ll}
x_{i}=a+i h, & 0 \leq i \leq N+1, \\
y_{j}=c+j k, & 0 \leq j \leq M+1 .
\end{array}
$$

Let us denote $u\left(x_{i}, y_{j}\right), r\left(x_{i}\right), p\left(x_{i}\right), q\left(x_{i}\right), f_{1}\left(y_{j}\right), f_{2}\left(x_{i}\right), g_{1}\left(y_{j}\right)$ and $g_{2}\left(x_{i}\right)$, by $U(i, j), r(i), p(i), q(i)$, $f_{1}(j), f_{2}(i), g_{1}(j)$ and $g_{2}(i)$ respectively.

We approximate the derivatives of elliptic Eq. (1) by the following finite differences of second-order

$$
\begin{aligned}
u_{y y}\left(x_{i}, y_{j}\right) & \approx \frac{U(i, j+1)-2 U(i, j)+U(i, j-1)}{k^{2}}, \\
{\left[p\left(x_{i}\right) u_{x}\left(x_{i}, y_{j}\right)\right]_{x} } & \approx \frac{p(i)[U(i+1, j)-U(i, j)]-p(i-1)[U(i, j)-U(i-1, j)]}{h^{2}},
\end{aligned}
$$

obtaining the explicit difference scheme

$$
\begin{aligned}
& r(i)[U(i, j+1)-2 U(i, j)+U(i, j-1)] \\
& \quad+\alpha^{2}\left[p(i) U(i+1, j)-\left(p(i)+p(i-1)+h^{2} q(i)\right) U(i, j)+p(i-1) U(i-1, j)\right]=0 \\
& 1 \leq i \leq N, \quad 1 \leq j \leq M
\end{aligned}
$$

where the parameter $\alpha$ was defined in (7).

Now we are going to study the consistency of constructed scheme (10) with Eq. (1), see [18], that is, guarantee that an exact solution of (1) is a good approximation of the numerical scheme (10) as the step-sizes $h$ and $k$ are sufficiently small. Under hypotheses given by (6), let us introduce the operators

$$
\Lambda[u]=r\left(x_{i}\right) u_{y y}\left(x_{i}, y_{j}\right)+\left[p\left(x_{i}\right) u_{x}\left(x_{i}, y_{j}\right)\right]_{x}-q\left(x_{i}\right) u\left(x_{i}, y_{j}\right),
$$

and

$$
\begin{aligned}
\Lambda_{h, k}[U]=r(i) & \frac{U(i, j+1)-2 U(i, j)+U(i, j-1)}{k^{2}} \\
& +\frac{p(i)[U(i+1, j)-U(i, j)]-p(i-1)[U(i, j)-U(i-1, j)]}{h^{2}}-q(i) U(i, j) .
\end{aligned}
$$

Let $\phi(x, y)$ be a twice differentiable solution of Eq. (1) and let us denote $\Phi(i, j)=\phi\left(x_{i}, y_{j}\right)$. The explicit scheme (10) is consistent with Eq. (1) if

$$
\lim _{h \rightarrow 0, k \rightarrow 0}\left(\Lambda[\phi]-\Lambda_{h, k}[\phi]\right)=0 .
$$

By (12) and considering the Taylor expansion of $\phi(\cdot, \cdot)$ about the point $\left(x_{i}, y_{j}\right)$ it follows that

$$
\begin{aligned}
\Lambda_{h, k}[\phi]=r(i)\left(\Phi_{y y}(i, j)+\mathrm{O}\left(k^{2}\right)\right)+ & \frac{1}{2}\left\{p(i)\left(\frac{2}{h} \Phi_{x}(i, j)+\Phi_{x x}(i, j)+\mathrm{O}\left(h^{2}\right)\right)\right. \\
& \left.-p(i-1)\left(\frac{2}{h} \Phi_{x}(i, j)-\Phi_{x x}(i, j)+\mathrm{O}\left(h^{2}\right)\right)\right\}-q(i) \Phi(i, j) .
\end{aligned}
$$

Note that by (11) one gets

$$
\begin{aligned}
\Lambda[\phi] & =r(i) \Phi_{y y}(i, j)+\left[p(i) \Phi_{x}(i, j)\right]_{x}-q(i) \Phi(i, j) \\
& =r(i) \Phi_{y y}(i, j)+p^{\prime}(i) \Phi_{x}(i, j)+p(i) \Phi_{x x}(i, j)-q(i) \Phi(i, j)
\end{aligned}
$$


Taking into account the Taylor expansion of $p(x)$ about $x_{i}$ one gets

$$
p(i-1)=p(i)-h p^{\prime}(i)+\mathrm{O}\left(h^{2}\right),
$$

and from (15)-(16) it follows that

$$
\begin{aligned}
\Lambda[\phi]-\Lambda_{h, k}[\phi]= & r(i) \Phi_{y y}(i, j)+p^{\prime}(i) \Phi_{x}(i, j)+p(i) \Phi_{x x}(i, j)-q(i) \Phi(i, j) \\
& \quad-r(i) \Phi_{y y}(i, j)-p(i) \Phi_{x x}(i, j)-p^{\prime}(i) \Phi_{x}(i, j)+\frac{h}{2} p^{\prime}(i) \Phi_{x x}(i, j) \\
& \quad+q(i) \Phi(i, j)+\mathrm{O}\left(h^{2}+k^{2}\right) \\
= & \frac{h}{2} p^{\prime}(i) \Phi_{x x}(i, j)+\mathrm{O}\left(h^{2}+k^{2}\right) .
\end{aligned}
$$

From (17) one gets (13) and thus the scheme (10) is consistent with Eq. (1), being the truncation error of order $O\left(h+k^{2}\right)$.

\section{Closed form numerical solution: Cases I-II}

Let us begin with the construction of the numerical solution for case I.

\section{Solution for Case I.}

The separation of discrete variables $i$ and $j$ allows to obtain solutions of the explicit scheme (10) of the form

$$
U^{I}(i, j)=H(i) G(j), \quad 1 \leq i \leq N, \quad 1 \leq j \leq M,
$$

where $H$ and $G$ are discrete functions to be determined. Substituting (18) into (10) one gets

$$
\begin{aligned}
& r(i)[G(j+1)-2 G(j)+G(j-1)] H(i) \\
& \quad+\alpha^{2}\left[p(i) H(i+1)-\left(p(i)+p(i-1)+h^{2} q(i)\right) H(i)+p(i-1) H(i-1)\right] G(j)=0 .
\end{aligned}
$$

By adding to both sides of (19) the term $\alpha^{2} \lambda r(i) H(i) G(j)$ being $\lambda$ a real number, it follows that

$$
\begin{aligned}
& r(i)\left[G(j+1)-\left(\alpha^{2} \lambda+2\right) G(j)+G(j-1)\right] H(i) \\
& \quad+\alpha^{2}\left[p(i) H(i+1)-\left(p(i)+p(i-1)+h^{2} q(i)-\lambda r(i)\right) H(i)+p(i-1) H(i-1)\right] G(j)=0 .
\end{aligned}
$$

Note that (20) holds true if

$$
-p(i) H(i+1)+\left(p(i)+p(i-1)+h^{2} q(i)\right) H(i)-p(i-1) H(i-1)=\lambda r(i) H(i), \quad 1 \leq i \leq N,
$$

and

$$
G(j+1)-\left(\alpha^{2} \lambda+2\right) G(j)+G(j-1)=0, \quad 1 \leq j \leq M .
$$

Under hypothesis (18), the boundary conditions from this case can be transformed in

$$
\begin{array}{rrl}
U^{I}(0, j)=0 ; & H(0) G(j)=0, & 0 \leq j \leq M+1, \\
U^{I}(N+1, j)=0 ; & H(N+1) G(j)=0, & 0 \leq j \leq M+1, \\
U^{I}(i, 0)=f_{2}(i) ; & H(i) G(0)=f_{2}(i), & 0 \leq i \leq N+1, \\
U^{I}(i, M+1)=0 ; & H(i) G(M+1)=0, & 0 \leq i \leq N+1 .
\end{array}
$$

Note by using (18), the boundary conditions (23)-(24) for $U^{I}(i, j)$ is granted if

$$
\begin{aligned}
H(0) & =0, \\
H(N+1) & =0 .
\end{aligned}
$$


Discrete Eq. (21) together with boundary conditions (27)-(28) define a discrete Sturm-Liouville problem having $N$ eigenpairs $\left(\lambda_{n},\left\{H_{n}(i): 1 \leq i \leq N\right\}\right)_{n=1}^{N}$ where the eigenfunctions $\left\{H_{n}(\cdot)\right\}_{n=1}^{N}$ are taken orthonormal with respect to the weight function $r(i)$, see [19, Chapter 11]. The eigenpairs of the Sturm-Liouville (21), (23)-(24) satisfy the matrix eigenvalue problem

$$
A H_{n}=\lambda_{n} R H_{n},
$$

where $A$ is the $N \times N$ tridiagonal and symmetric matrix

$$
A=\left(\begin{array}{cccccc}
s(1) & -p(1) & 0 & \cdots & \cdots & 0 \\
-p(1) & s(2) & -p(2) & & & \vdots \\
0 & \ddots & \ddots & \ddots & & \vdots \\
\vdots & & \ddots & \ddots & \ddots & 0 \\
\vdots & & & \ddots & s(N-1) & -p(N-1) \\
0 & \ldots & \ldots & 0 & -p(N-1) & s(N)
\end{array}\right)
$$

with

$$
s(i)=p(i)+p(i-1)+h^{2} q(i), \quad 1 \leq i \leq N,
$$

and $R$ is the $N \times N$ diagonal matrix

$$
R=\operatorname{diag}(r(1), r(2), \ldots, r(N)) .
$$

Taking into account that by definition, the elliptic Eq. (1) verifies

$$
\operatorname{sig}(p(x))=\operatorname{sig}(r(x)), \quad x \in[a, b],
$$

and imposing

$$
\begin{aligned}
& p(i)>0, \quad r(i)>0, \quad 0 \leq i \leq N+1, \\
& q(i)>0, \quad 0 \leq i \leq N+1,
\end{aligned}
$$

we show that real symmetric matrix $A$ defined by (30) is positive definite, i.e. the quadratic form defined by $A$ verifies

$$
X^{T} A X>0, \quad \forall X \in \mathbb{R}^{N} \text { non zero. }
$$

In fact, developing the expression of (36) and taking into account (34)-(35), we obtain

$$
\begin{aligned}
X^{T} A X= & {\left[p(1)+p(0)+h^{2} q(1)\right] X^{2}(1)+\left[p(N)+p(N-1)+h^{2} q(N)\right] X^{2}(N) } \\
& +\sum_{\ell=2}^{N-1}\left[p(\ell)+p(\ell-1)+h^{2} q(\ell)\right] X^{2}(\ell)-2 \sum_{\ell=1}^{N-1} p(\ell) X(\ell) X(\ell+1) \\
= & p(0) X^{2}(1)+p(N) X^{2}(N)+\sum_{\ell=1}^{N} h^{2} q(\ell) X^{2}(\ell)+\sum_{\ell=1}^{N-1} p(\ell)[X(\ell)-X(\ell-1)]^{2}>0 .
\end{aligned}
$$

Then one gets that

$$
\lambda_{n}>0, \quad \forall n=1, \ldots, N
$$

being $\left\{\lambda_{n}\right\}_{n=1}^{N}$ the eigenvalues of matrix eigenvalue problem (29).

Note that by (32) and (34) matrix $R$ is invertible, and from (29) the eigenpairs $\left(\lambda_{n}, H_{n}(\cdot)\right)_{n=1}^{N}$ can be obtained as the eigenpairs of the following algebraic eigenvalue problem

$$
R^{-1} A H_{n}=\lambda_{n} H_{n}, \quad 1 \leq n \leq N .
$$


By Amir-Moez inequality, see [20, p. 246], the eigenvalues $\left\{\lambda_{n}\right\}_{n=1}^{N}$ of $R^{-1} A$ are all positive. In the practise, the eigenpairs of (39) can be computed, for example, using the MATLAB software .

Once the $N$ eigenvalues $\left\{\lambda_{n}\right\}$ of (39) have been determined we are going to solve the $N$ problems obtained from (22), (25)-(26):

$$
\begin{aligned}
G_{n}(j+1)-\left(\alpha^{2} \lambda_{n}+2\right) G_{n}(j)+G_{n}(j-1)=0, & 1 \leq j \leq M, \\
\sum_{n=1}^{N} H_{n}(i) G_{n}(0)=f_{2}(i), & j=0, \\
\sum_{n=1}^{N} H_{n}(i) G_{n}(M+1)=0, & j=M+1 .
\end{aligned}
$$

Given a fixed value $n$, the characteristic equation associated to (40) is

$$
z_{n}^{2}-\left(\alpha^{2} \lambda_{n}+2\right) z_{n}+1=0,
$$

which has two simple real roots

$$
z_{1, n}=\frac{\alpha^{2} \lambda_{n}+2+\sqrt{\left(\alpha^{2} \lambda_{n}+2\right)^{2}-4}}{2}, \quad z_{2, n}=\frac{\alpha^{2} \lambda_{n}+2-\sqrt{\left(\alpha^{2} \lambda_{n}+2\right)^{2}-4}}{2}
$$

because of the discriminant of (44) takes the value $\Delta=\left(\alpha^{2} \lambda_{n}+2\right)^{2}-4>0$ since $\lambda_{n}>0$. Using the Cardano-Vieta relationship, $m_{1} \cdot m_{2}=c / a$, for a second-order equations $a x^{2}+b x+c=0$ being $m_{1}$ and $m_{2}$ two simple reals roots, we obtain $z_{1, n} \cdot z_{2, n}=1$ or equivalently

$$
z_{2, n}=\frac{1}{z_{1, n}} \quad \text { or } \quad z_{1, n}=\frac{1}{z_{2, n}} .
$$

Furthermore, it is easy prove

$$
z_{1, n}>1, \quad z_{2, n}>0
$$

then from (45)-(46) one gets

$$
0<z_{2, n}<1 .
$$

As Eq. (40) is linear in $G_{n}$ its general solution, given a fixed value $n, 1 \leq n \leq N$, is

$$
G_{n}(j)=c_{n}^{I} \cdot z_{1, n}^{j}+d_{n}^{I} \cdot z_{2, n}^{j}, \quad 1 \leq j \leq M,
$$

being $c_{n}^{I}$ and $d_{n}^{I}$ real constants to be determined. From linearity, by superposition of the $N$ solutions of (10) of the form (18) yields

$$
\mathbf{U}^{I}(i, j)=\sum_{n=1}^{N} U_{n}^{I}(i, j)=\sum_{n=1}^{N} H_{n}(i) G_{n}(j), \quad 1 \leq i \leq N, \quad 1 \leq j \leq M .
$$

In order to determine the constants $c_{n}^{I}$ and $d_{n}^{I}$ in (48) we impose the boundary conditions (41) and (42). Using (49), the boundary condition $(26)$ for $\mathbf{U}^{I}(i, j)$ is granted if

$$
G_{n}(M+1)=0, \quad 1 \leq n \leq N .
$$

Taking $j=M+1$ in (48), and using (50) and property (45) one gets

$$
\begin{aligned}
& 0=G_{n}(M+1)=c_{n}^{I} z_{1, n}^{M+1}+d_{n}^{I} z_{2, n}^{M+1} ; \\
& d_{n}^{I}=-c_{n}^{I}\left(\frac{z_{1, n}}{z_{2, n}}\right)^{M+1}=-c_{n}^{I}\left(\frac{z_{1, n}}{1 / z_{1, n}}\right)^{M+1}=-c_{n}^{I} z_{1, n}^{2 M+2} .
\end{aligned}
$$


In view of (51), expression (48) can be rewritten in the form

$$
G_{n}(j)=c_{n}^{I}\left(z_{1, n}^{j}-z_{1, n}^{2 M+2} z_{2, n}^{j}\right) .
$$

Substituting (52) in (49) one gets

$$
\mathbf{U}^{I}(i, j)=\sum_{n=1}^{N} H_{n}(i) c_{n}^{I}\left(z_{1, n}^{j}-z_{1, n}^{2 M+2} z_{2, n}^{j}\right) \quad 1 \leq i \leq N, \quad 1 \leq j \leq M .
$$

Finally, in order to determine constants $c_{n}^{I}$ in (53), we use the boundary condition (25) for $j=0$ obtaining from (53)

$$
f_{2}(i)=\mathbf{U}^{I}(i, 0)=\sum_{n=1}^{N} H_{n}(i) G_{n}(0)=\sum_{n=1}^{N} H_{n}(i) c_{n}^{I}\left(1-z_{1, n}^{2 M+2}\right) .
$$

Constants $c_{n}^{I}, 1 \leq n \leq N$, can be determined from (54) premultiplying in both sides by $r(i) H_{\ell}(i)$, $1 \leq \ell \leq N$; adding the obtained result from $i=1$ up to $i=N$; and using the orthonormality of eigenfunctions $\left\{H_{n}(i)\right\}_{n=1}^{N}$ with respect to the weight function $r(i)$. Hence one gets

$$
\begin{aligned}
\sum_{i=1}^{N} r(i) H_{\ell}(i) f_{2}(i) & =\sum_{n=1}^{N} c_{n}^{I}\left(1-z_{1, n}^{2 M+2}\right)\left(\sum_{i=1}^{N} r(i) H_{\ell}(i) H_{n}(i)\right) \\
\stackrel{(n=\ell)}{=} & c_{\ell}^{I}\left(1-z_{1, \ell}^{2 M+2}\right) \sum_{i=1}^{N} r(i) H_{\ell}^{2}(i)=c_{\ell}^{I}\left(1-z_{1, \ell}^{2 M+2}\right) .
\end{aligned}
$$

From (55), constants $c_{n}^{I}, 1 \leq n \leq N$, take the form

$$
c_{n}^{I}=\frac{1}{1-z_{1, n}^{2 M+2}} \sum_{\sigma=1}^{N} r(\sigma) H_{n}(\sigma) f_{2}(\sigma), \quad 1 \leq n \leq N .
$$

Then we have constructed a closed form numerical solution of (1) with the boundary conditions of type I, given by (39), (44), (53) and (56).

\section{Solution for Case II.}

For the sake of brevity in the presentation and from the similarity of the first two boundary conditions of cases I and II, and taking into account the developments made in case I, we seek solutions of the form

$$
\mathbf{U}^{I I}(i, j)=\sum_{n=1}^{N} H_{n}(i) G_{n}^{I I}(j), \quad 1 \leq i \leq N, \quad 1 \leq j \leq M,
$$

being $\left\{H_{n}(\cdot)\right\}_{n=1}^{N}$ the orthonormal eigenfunctions computed for the problem (39) as in case I, because we have in this case the same boundary conditions on the left and right sides: $u(a, y)=u(b, y)=0$. Furthermore, functions $G_{n}^{I I}(j)$ are the same type as in case I:

$$
G_{n}^{I I}(j)=c_{n}^{I I} \cdot z_{1, n}^{j}+d_{n}^{I I} \cdot z_{2, n}^{j}, \quad 1 \leq n \leq N,
$$

being $c_{n}^{I I}$ and $d_{n}^{I I}$ real constants to be determined, and $z_{1, n}$ and $z_{2, n}$ the roots computed in case I given by (44). In order to determinate the constants $c_{n}^{I I}$ and $d_{n}^{I I}$, we need to use the boundary conditions $u(x, c)=0$ and $u(x, d)=g_{2}(x)$, which by (57) take the form

$$
\begin{aligned}
0=\mathbf{U}^{\mathbf{I I}}(i, 0)=\sum_{n=1}^{N} H_{n}(i) G_{n}^{I I}(0), & 0 \leq i \leq N+1, \\
g_{2}(i)=\mathbf{U}^{\mathbf{I I}}(i, M+1)=\sum_{n=1}^{N} H_{n}(i) G_{n}^{I I}(M+1), & 0 \leq i \leq N+1 .
\end{aligned}
$$


Note that under condition

$$
G_{n}^{I I}(0)=0, \quad 1 \leq n \leq N,
$$

$\mathbf{U}^{I I}(i, j)$ satisfies $\mathbf{U}^{I I}(i, 0)=0$. Taking $j=0$ in (58) and using (61) one gets

$$
0=G_{n}^{I I}(0)=c_{n}^{I I}+d_{n}^{I I} ; \quad d_{n}^{I I}=-c_{n}^{I I} .
$$

Substituting (62) in (58) we obtain

$$
G_{n}^{I I}(j)=c_{n}^{I I}\left(z_{1, n}^{j}-z_{2, n}^{j}\right), \quad 1 \leq j \leq M,
$$

and using (63) in (57) one gets

$$
\mathbf{U}^{I I}(i, j)=\sum_{n=1}^{N} H_{n}(i) c_{n}^{I I}\left(z_{1, n}^{j}-z_{2, n}^{j}\right) \quad 1 \leq i \leq N, \quad 1 \leq j \leq M .
$$

Finally, in order to determine constants $c_{n}^{I I}$ in (64), we use the boundary condition (60) for $j=M+1$ which by (63) is transformed in

$$
g_{2}(i)=\sum_{n=1}^{N} H_{n}(i) c_{n}^{I I}\left(z_{1, n}^{M+1}-z_{2, n}^{M+1}\right) .
$$

Operating as in previous case I (see (55)), constants $c_{n}^{I I}$ take the form

$$
c_{n}^{I I}=\frac{1}{z_{1, n}^{M+1}-z_{2, n}^{M+1}} \sum_{\sigma=1}^{N} r(\sigma) H_{n}(\sigma) g_{2}(\sigma), \quad 1 \leq n \leq N .
$$

Thus we have constructed a closed form numerical solution of (1) with the boundary conditions of type II, given by (39), (44), (64) and (66).

\section{Solution for Cases I and II.}

By the linearity of Eq. (1) and the superposition principle, the constructed closed form numerical solution for both cases I-II is

$$
\mathbf{U}^{I}(i, j)+\mathbf{U}^{I I}(i, j)=\sum_{n=1}^{N} H_{n}(i)\left[c_{n}^{I}\left(z_{1, n}^{j}-z_{1, n}^{2 M+2} z_{2, n}^{j}\right)+c_{n}^{I I}\left(z_{1, n}^{j}-z_{2, n}^{j}\right)\right],
$$

being $\left\{H_{n}(\cdot)\right\}_{n=1}^{N}$ the orthonormal eigenfunctions of problem (39) and the real constants $z_{1, n}, z_{2, n}$, $c_{n}^{I}$ and $c_{n}^{I I}$ defined by (44), (56) and (66), respectively.

\section{Closed form numerical solution: Cases III-IV}

Solution for Case III.

By separation of discrete variables $i$ and $j$, now let us seek solutions of the explicit scheme (10) of the form

$$
U^{\mathrm{III}}(i, j)=H^{\mathrm{III}}(j) G^{\mathrm{III}}(i), \quad 1 \leq i \leq N, \quad 1 \leq j \leq M .
$$

Under hypothesis (68) the boundary conditions of this case can be transformed in

$$
\begin{aligned}
U^{\mathrm{III}}(0, j) & =H^{\mathrm{III}}(j) G^{\mathrm{III}}(0)=f_{1}(j), & & 0 \leq j \leq M+1, \\
U^{\mathrm{III}}(N+1, j) & =H^{\mathrm{III}}(j) G^{\mathrm{III}}(N+1)=0, & & 0 \leq j \leq M+1, \\
U^{\mathrm{III}}(i, 0) & =H^{\mathrm{III}}(0) G^{\mathrm{III}}(i)=0, & & 0 \leq i \leq N+1, \\
U^{\mathrm{III}}(i, M+1) & =H^{\mathrm{III}}(M+1) G^{\mathrm{III}}(i)=0, & & 0 \leq i \leq N+1 .
\end{aligned}
$$


By imposing to $U^{\mathrm{III}}(i, j)$, given by (68), that satisfies (10) and by adding to both sides of (10) the term $\alpha^{2} \hat{\lambda} r(i) H^{\mathrm{III}}(j) G^{\mathrm{III}}(i)$ where $\hat{\lambda}$ is a real number to be determined and the real number $\alpha$ was defined in (7), one gets

$$
\begin{aligned}
& r(i)\left[H^{\mathrm{III}}(j+1)-\left(\alpha^{2} \hat{\lambda}+2\right) H^{\mathrm{III}}(j)+H^{\mathrm{III}}(j-1)\right] G^{\mathrm{III}}(i) \\
+ & \alpha^{2}\left[p(i) G^{\mathrm{III}}(i+1)-\left(p(i)+p(i-1)+h^{2} q(i)-\hat{\lambda} r(i)\right) G^{\mathrm{III}}(i)+p(i-1) G^{\mathrm{III}}(i-1)\right] H^{\mathrm{III}}(j)=0 .
\end{aligned}
$$

Note that (73) holds true if

$$
H^{\mathrm{III}}(j+1)-\left(\alpha^{2} \hat{\lambda}+2\right) H^{\mathrm{III}}(j)+H^{\mathrm{III}}(j-1)=0, \quad 1 \leq j \leq M,
$$

and

$$
p(i) G^{\mathrm{II}}(i+1)-\left(p(i)+p(i-1)+h^{2} q(i)-\hat{\lambda} r(i)\right) G^{\mathrm{III}}(i)+p(i-1) G^{\mathrm{III}}(i-1)=0, \quad 1 \leq i \leq N .
$$

By using (68), the boundary conditions (71)-(72) for $U^{\mathrm{III}}(i, j)$ are granted if

$$
\begin{aligned}
H^{\mathrm{III}}(0) & =0, \\
H^{\mathrm{III}}(M+1) & =0 .
\end{aligned}
$$

Discrete Eq. (74) together with boundary conditions (76)-(77) define a discrete Sturm-Liouville problem having $M$ eigenpairs $\left(\hat{\lambda}_{m},\left\{H_{m}^{\mathrm{III}}(j): 1 \leq j \leq M\right\}\right)_{m=1}^{M}$ to be determined and which satisfies the algebraic eigenvalue problem:

$$
\frac{1}{\alpha^{2}} \tilde{A} H_{m}^{\mathrm{III}}=\hat{\lambda}_{m} H_{m}^{\mathrm{III}},
$$

where the real number $\alpha$ was defined in (7) and $\tilde{A}$ is the $M \times M$ symmetric tridiagonal matrix with its constant entries:

$$
\tilde{A}=\left(\begin{array}{cccccc}
-2 & 1 & 0 & \cdots & \cdots & 0 \\
1 & -2 & 1 & & & \vdots \\
0 & \ddots & \ddots & \ddots & & \vdots \\
\vdots & & \ddots & \ddots & \ddots & 0 \\
\vdots & & & \ddots & -2 & 1 \\
0 & \cdots & \cdots & 0 & 1 & -2
\end{array}\right)
$$

We show now that the symmetric matrix $\frac{1}{\alpha^{2}} \tilde{A}$ is negative definite, i.e.

$$
\frac{1}{\alpha^{2}}\left(X^{T} \tilde{A} X\right)<0, \quad \forall X \in \mathbb{R}^{M} \text { non zero. }
$$

In fact, developing the expression (80) and taking into account that the parameter $\alpha$ is positive, we obtain

$$
\begin{aligned}
\frac{1}{\alpha^{2}}\left(X^{T} \tilde{A} X\right)= & \frac{1}{\alpha^{2}}\left(-2 X^{2}(1)-2 X^{2}(M)-2 \sum_{\ell=2}^{M-1} X^{2}(\ell)-\sum_{\ell=1}^{M-1}[X(\ell)-X(\ell+1)]^{2}\right. \\
& \left.+X^{2}(1)+X^{2}(M)+2 \sum_{\ell=2}^{M-1} X^{2}(\ell)\right) \\
= & -\frac{1}{\alpha^{2}}\left(X^{2}(1)+X^{2}(M)+\sum_{\ell=1}^{M-1}[X(\ell)-X(\ell+1)]^{2}\right)<0 .
\end{aligned}
$$


Thus, from (81) one gets that

$$
\hat{\lambda}_{m}<0, \quad \forall m=1, \ldots, M,
$$

being $\tilde{\lambda}_{m}$ the eigenvalues of algebraic eigenvalue problem (78). This previous study, about the sign of eigenvalues of problem (78), is necessary in order to determine what kind of solutions can be obtained for Eq. (74). Thus, given a fixed value $m$, we consider the characteristic equation associated to (74):

$$
\hat{z}_{m}^{2}-\left(\alpha^{2} \hat{\lambda}_{m}+2\right) \hat{z}_{m}+1=0
$$

whose discriminant takes the value

$$
\Delta=\hat{\lambda}_{m} \alpha^{2}\left(\frac{\hat{\lambda}_{m} \alpha^{2}}{4}+1\right) .
$$

From (82) and under condition

$$
\alpha^{2}<\frac{4}{\left|\hat{\lambda}_{m}\right|}, \quad \forall m=1, \ldots, M,
$$

one gets $\Delta>0$. Condition (85) is satisfied taking $\alpha=k / h$ small enough, so that

$$
\alpha<\frac{2}{\sqrt{\left|\hat{\lambda}_{\max }\right|}}, \quad \hat{\lambda}_{\max }=\max \left\{\hat{\lambda}_{1}, \hat{\lambda}_{2}, \ldots, \hat{\lambda}_{M}\right\} .
$$

By (84), Eq. (83) has two conjugate complex roots:

$$
\begin{aligned}
& \hat{z}_{1, m}=1+\frac{\hat{\lambda}_{m} \alpha^{2}}{2}+\hat{i} \sqrt{1-\left(1+\frac{\hat{\lambda}_{m} \alpha^{2}}{2}\right)^{2}}=\left|\hat{z}_{1, m}\right| \exp \left(\hat{i} \theta_{m}\right)=\exp \left(\hat{i} \theta_{m}\right), \\
& \hat{z}_{2, m}=1+\frac{\hat{\lambda}_{m} \alpha^{2}}{2}-\hat{i} \sqrt{1-\left(1+\frac{\hat{\lambda}_{m} \alpha^{2}}{2}\right)^{2}}=\left|\hat{z}_{2, m}\right| \exp \left(-\hat{i} \theta_{m}\right)=\exp \left(-\hat{i} \theta_{m}\right),
\end{aligned}
$$

with

$$
0<\theta_{m}<\pi, \quad \cos \left(\theta_{m}\right)=1+\frac{\hat{\lambda}_{m} \alpha^{2}}{2}, \quad \hat{i}=\sqrt{(-1)} .
$$

Note that from (87)-(88) we obtain

$$
\left(\hat{z}_{1, m}\right)^{j}=\cos \left(j \theta_{m}\right)+\hat{i} \sin \left(j \theta_{m}\right), \quad\left(\hat{z}_{2, m}\right)^{j}=\cos \left(j \theta_{m}\right)-\hat{i} \sin \left(j \theta_{m}\right), \quad 1 \leq j \leq M .
$$

In view of (90), the general solutions of Eq. (74) can be written in the form

$$
\begin{array}{r}
H_{m}^{\mathrm{III}}(j)=\tilde{C}_{m}\left(\hat{z}_{1, m}\right)^{j}+\tilde{D}_{m}\left(\hat{z}_{2, m}\right)^{j}=C_{m} \cos \left(j \theta_{m}\right)+D_{m} \sin \left(j \theta_{m}\right), \\
1 \leq j \leq M, \quad C_{m}, D_{m} \in \mathbb{R} .
\end{array}
$$

In order to determine the real constants $C_{m}, D_{m}$ and the angles $\theta_{m}$, we use the boundary conditions (76)-(77). Thus, substituting the boundary condition (76) in (91) for $j=0$ we obtain $C_{m}=0$ and from (91) one gets the expression

$$
H_{m}^{\mathrm{III}}(j)=D_{m} \sin \left(j \theta_{m}\right), \quad 1 \leq j \leq M .
$$

Substituting the boundary condition (77) in (92) for $j=M+1$ and avoiding non trivial solutions one gets $0=D_{m} \sin \left((M+1) \theta_{m}\right)$, or equivalently

$$
\theta_{m}=\left(\frac{\pi}{M+1}\right) m, \quad m=1, \ldots, M .
$$


Taking $D_{m}=1, \forall m$, by (92)-(93) we have determined the $M$ eigenfunctions of Sturm-Liouville problem (74), (76)-(77) given by

$$
H_{m}^{\mathrm{III}}(j)=\sin \left(\frac{j m}{M+1} \pi\right), \quad 1 \leq j \leq M, \quad m=1, \ldots, M,
$$

which are orthogonal (with respect to the weight constant function 1). Furthermore, by (82), (89) and (93) the $M$ eigenvalues of Sturm-Liouville problem (74), (76)-(77) take the negative values

$$
\hat{\lambda}_{m}=\frac{2}{\alpha^{2}}\left(\cos \left(\frac{m}{M+1} \pi\right)-1\right), \quad m=1, \ldots, M
$$

Note that in view of (95), the condition (86) for the parameter $\alpha$ always is verified. From linearity, by superposition of the $M$ solutions of (10) of the form (68) it yields

$$
\begin{array}{r}
\mathbf{U}^{\mathrm{III}}(i, j)=\sum_{m=1}^{M} U_{m}^{\mathrm{III}}(i, j)=\sum_{m=1}^{M} H_{m}^{\mathrm{III}}(j) G_{m}^{\mathrm{III}}(i)=\sum_{m=1}^{M} \sin \left(\frac{j m}{M+1} \pi\right) G_{m}^{\mathrm{III}}(i), \\
1 \leq i \leq N, \quad 1 \leq j \leq M .
\end{array}
$$

The $M$ obtained eigenvalues $\left\{\hat{\lambda}_{m}\right\}$, given by (95), generate the following $M$ problems obtained from (75), (69)-(70):

$$
\begin{array}{r}
p(i) G_{m}^{\mathrm{III}}(i+1)-\left(p(i)+p(i-1)+h^{2} q(i)-\hat{\lambda}_{m} r(i)\right) G_{m}^{\mathrm{III}}(i)+p(i-1) G_{m}^{\mathrm{III}}(i-1)=0, \quad 1 \leq i \leq N, \\
(97) \\
\sum_{m=1}^{M} H_{m}^{\mathrm{III}}(j) G_{m}^{\mathrm{III}}(0)=f_{1}(j), \quad i=0,(98) \\
\sum_{m=1}^{M} H_{m}^{\mathrm{III}}(j) G_{m}^{\mathrm{III}}(N+1)=0, \quad i=N+1 .
\end{array}
$$

The boundary condition (98) can be transformed in the following boundary condition by using: expression (96), the orthogonality of the eigenfunctions $\left\{H_{m}^{\mathrm{III}}(\cdot)\right\}$ given by $(94)$, and the argument described in case I (see (55)):

$$
G_{m}^{\mathrm{III}}(0)=\frac{\sum_{\sigma=1}^{M} H_{m}^{\mathrm{III}}(\sigma) f_{1}(\sigma)}{\sum_{\sigma=1}^{M}\left(H_{m}^{\mathrm{III}}(\sigma)\right)^{2}}, \quad 1 \leq m \leq M .
$$

If the eigenfunctions $\left\{H_{m}^{\mathrm{III}}(j)\right\}$ are taken orthonormal, then the boundary conditions (100) are rewritten in the form

$$
G_{m}^{\mathrm{III}}(0)=\sum_{\sigma=1}^{M} \tilde{H}_{m}^{\mathrm{III}}(\sigma) f_{1}(\sigma):=\tilde{a}_{m}, \quad 1 \leq m \leq M
$$

On the other hand, using (96), the boundary condition (99) for $\mathbf{U}^{\mathrm{III}}(i, j)$ is granted if

$$
G_{m}^{\mathrm{III}}(N+1)=0
$$

Taking into account hypothesis (34), we can rewrite the discrete variable coefficient linear secondorder Eq. (97) as follow:

$$
G_{m}^{\mathrm{III}}(i+1)=-\frac{a_{1, m}(i)}{a_{2}(i)} G_{m}^{\mathrm{III}}(i)-\frac{a_{0}(i)}{a_{2}(i)} G_{m}^{\mathrm{III}}(i-1), \quad 1 \leq i \leq N, \quad 1 \leq m \leq M,
$$


with the notation

$$
\left.\begin{array}{l}
a_{0}(i):=p(i-1) \\
a_{1, m}(i):=-p(i)-p(i-1)-h^{2} q(i)+\hat{\lambda}_{m} r(i) \\
a_{2}(i):=p(i)
\end{array}\right\}
$$

where $a_{1, m}(\cdot)$ denotes that the coefficient $a_{1}$ varies for each $m, 1 \leq m \leq M$. Thus, we are going to resolve the $M$ boundary problems (103)-(104) with the transformed boundary conditions (101)-(102). Taking the following variable changes

$$
L_{1, m}(i-1):=G_{m}^{\mathrm{III}}(i-1), \quad L_{2, m}(i-1):=G_{m}^{\mathrm{III}}(i)
$$

the $M$ linear second-order boundary problems (103)-(104), (101)-(102) can be rewritten as the $M$ linear first-order systems

$$
\left.\begin{array}{l}
L_{1, m}(i)=L_{2, m}(i-1) \\
L_{2, m}(i)=-\frac{a_{1, m}(i)}{a_{2}(i)} L_{2, m}(i-1)-\frac{a_{0}(i)}{a_{2}(i)} L_{1, m}(i-1)
\end{array}\right\}, \quad 1 \leq m \leq M,
$$

with the boundary conditions

$$
\begin{gathered}
L_{1, m}(0)=\tilde{a}_{m}, \\
L_{1, m}(N+1)=0,
\end{gathered}
$$

being $\tilde{a}_{m}$ defined by (101). The system (106) can be rewritten in a matrix form, for each $m$, as follow

$$
\vec{L}_{m}(i)=\tilde{A}_{m}(i) \vec{L}_{m}(i-1), \quad 1 \leq i \leq N, \quad 1 \leq m \leq M,
$$

denoting by $\vec{L}_{m}(\cdot)$ the $2 \times 1$ vectors

$$
\vec{L}_{m}(\cdot)=\left[\begin{array}{c}
L_{1, m}(\cdot) \\
L_{2, m}(\cdot)
\end{array}\right], \quad 1 \leq m \leq M
$$

and $\tilde{A}_{m}(\cdot)$ the $2 \times 2$ matrices

$$
\tilde{A}_{m}(\cdot)=\left[\begin{array}{cc}
0 & 1 \\
-\frac{a_{0}(\cdot)}{a_{2}(\cdot)} & -\frac{a_{1, m}(\cdot)}{a_{2}(\cdot)}
\end{array}\right], \quad 1 \leq m \leq M,
$$

see $\left[19\right.$, Chapter 1]. Note that, under condition (34), the matrix $\tilde{A}_{m}(\cdot)$, defined by (111), satisfies for each $m$ :

$$
\tilde{A}_{m}(\cdot) \text { is an invertible matrix }, \quad 1 \leq m \leq M,
$$

because of

$$
\operatorname{det}\left(\tilde{A}_{m}(i)\right)=\frac{a_{0}(i)}{a_{2}(i)}=\frac{p(i-1)}{p(i)} \neq 0, \quad \forall i, \quad 0 \leq i \leq N+1 .
$$

For the sake of convenience, problem (109) with boundary condition (108), is solved by backward recurrence from $i=N$ up to $i=1$. For this goal, we consider the following equivalent expression to (109) which is obtained from (109) and (112):

$$
\vec{L}_{m}(i)=\tilde{A}_{m}^{-1}(i+1) \vec{L}_{m}(i+1), \quad 1 \leq i \leq N,
$$

being

$$
\tilde{A}_{m}^{-1}(\cdot)=\left[\begin{array}{cc}
-\frac{a_{1, m}(\cdot)}{a_{0}(\cdot)} & -\frac{a_{2}(\cdot)}{a_{0}(\cdot)} \\
1 & 0
\end{array}\right]
$$


By (114) for $i=N$ up to $i=1$, we obtain

$$
\begin{aligned}
& \vec{L}_{m}(N)=\tilde{A}_{m}^{-1}(N+1) \vec{L}_{m}(N+1), \\
& \vec{L}_{m}(N-1)=\tilde{A}_{m}^{-1}(N) \vec{L}_{m}(N)=\tilde{A}_{m}^{-1}(N) \tilde{A}_{m}^{-1}(N+1) \vec{L}_{m}(N+1)=\prod_{\ell=N}^{N+1} \tilde{A}_{m}^{-1}(\ell) \vec{L}_{m}(N+1), \\
& \vec{L}_{m}(N-2)=\tilde{A}_{m}^{-1}(N-1) \vec{L}_{m}(N-1)=\prod_{\ell=N-1}^{N+1} \tilde{A}_{m}^{-1}(\ell) \vec{L}_{m}(N+1), \\
& \vec{L}_{m}(N-3)=\tilde{A}_{m}^{-1}(N-2) \vec{L}_{m}(N-2)=\prod_{\ell=N-2}^{N+1} \tilde{A}_{m}^{-1}(\ell) \vec{L}_{m}(N+1), \\
& \ldots \\
& \vec{L}_{m}(1)=\tilde{A}_{m}^{-1}(2) \vec{L}_{m}(2)=\prod_{\ell=2}^{N+1} \tilde{A}_{m}^{-1}(\ell) \vec{L}_{m}(N+1),
\end{aligned}
$$

that is, the solution of Eq. (114), for each $m, 1 \leq m \leq M$, takes the form

$$
\vec{L}_{m}(i)=\prod_{\ell=i+1}^{N+1} \tilde{A}_{m}^{-1}(\ell) \vec{L}_{m}(N+1)=\prod_{\ell=i+1}^{N+1} \tilde{A}_{m}^{-1}(\ell)\left[\begin{array}{c}
0 \\
L_{2, m}(N+1)
\end{array}\right], \quad 1 \leq i \leq N
$$

where $w=L_{2, m}(N+1)$ is a free component. Our task is to choose this free component properly to generate a solution of problem (106)-(108). Let us take $w=1$ and

$$
\vec{Y}_{m}(i)=\prod_{\ell=i+1}^{N+1} \tilde{A}_{m}^{-1}(\ell)\left[\begin{array}{l}
0 \\
1
\end{array}\right], \quad 0 \leq i \leq N .
$$

Note that by construction its first component $[1,0] \vec{Y}_{m}(i)$ satisfies (108). Following the shooting method ideas, and taking into account that from (112) one gets

$$
\vec{v}_{m}:=\vec{Y}_{m}(0) \neq \mathbf{0}
$$

and provided that $[1,0] \vec{v}_{m} \neq 0$, then

$$
L_{1, m}(i)=\frac{\tilde{a}_{m}}{[1,0] \vec{v}_{m}}[1,0] \vec{Y}_{m}(i), \quad 0 \leq i \leq N+1,
$$

satisfies (107)-(109), for each $m, 1 \leq m \leq M$. Finally, undoing the variable change $L_{1, m}(i):=G_{m}^{\mathrm{III}}(i)$ and by (96) we have constructed a solution for the case III:

$$
\mathbf{U}^{\mathrm{III}}(i, j)=\sum_{m=1}^{M} \tilde{H}_{m}^{\mathrm{III}}(j) L_{1, m}(i), \quad 1 \leq i \leq N, \quad 1 \leq j \leq M,
$$

where $\left\{\tilde{H}_{m}^{\mathrm{III}(\cdot)}\right\}_{m=1}^{M}$ are the orthonormal eigenfunctions obtained from the eigenfunctions $\left\{H_{m}^{\mathrm{III}}(\cdot)\right\}_{m=1}^{M}$ given by (94), and $\left\{L_{1, m}(\cdot)\right\}_{m=1}^{M}$ are obtained from (117)-(119).

\section{Solution for Case IV.}

From the similarity of the boundary conditions of cases III and IV on up and down sides: $u(x, c)=$ $u(x, d)=0$, and taking into account the developments made in case III, we seek solutions of the form

$$
\mathbf{U}^{\mathrm{IV}}(i, j)=\sum_{m=1}^{M} \tilde{H}_{m}^{\mathrm{III}}(j) G_{m}^{\mathrm{IV}}(i), \quad 1 \leq i \leq N, \quad 1 \leq j \leq M,
$$


being the eigenfunctions $\left\{\tilde{H}_{m}^{\mathrm{III}}(\cdot)\right\}_{m=1}^{M}$ those obtained from orthonormalizing the eigenfunctions $\left\{H_{m}^{\mathrm{nII}}(\cdot)\right\}_{m=1}^{M}$ of case III given by (94) and computed for the problem (74), (76) and (77). Furthermore, as in case III, the functions $G_{m}^{\mathrm{IV}}(i)$ are the solutions of the following $M$ boundary value problems:

$$
\begin{aligned}
G_{m}^{\mathrm{IV}}(i+1) & =-\frac{a_{1, m}(i)}{a_{2}(i)} G_{m}^{\mathrm{IV}}(i)-\frac{a_{0}(i)}{a_{2}(i)} G_{m}^{\mathrm{IV}}(i-1), \quad 1 \leq i \leq N, \quad 1 \leq m \leq M, \\
G_{m}^{\mathrm{IV}}(0) & =0 \\
G_{m}^{\mathrm{IV}}(N+1) & =\sum_{\sigma=1}^{M} \tilde{H}_{m}^{\mathrm{III}}(\sigma) g_{1}(\sigma):=\tilde{b}_{m},
\end{aligned}
$$

where the constants $a_{0}(i), a_{1, m}(i)$ and $a_{2}(i)$ where defined in (104).

In a similar way as in case III (see from (105)-(111)), we consider the discrete variable changes

$$
T_{1, m}(i-1):=G_{m}^{\mathrm{IV}}(i-1), \quad T_{2, m}(i-1):=G_{m}^{\mathrm{IV}}(i),
$$

Let us denote by $\vec{T}_{m}(\cdot)$ the $2 \times 1$ vectors

$$
\vec{T}_{m}(\cdot)=\left[\begin{array}{c}
T_{1, m}(\cdot) \\
T_{2, m}(\cdot)
\end{array}\right], \quad 1 \leq m \leq M
$$

then from (122)-(124) we obtain the following $M$ linear first-order systems in a matrix form with the boundary conditions

$$
\begin{aligned}
\vec{T}_{m}(i) & =\tilde{A}_{m}(i) \vec{T}_{m}(i-1), \quad 1 \leq i \leq N, \quad 1 \leq m \leq M, \\
T_{1, m}(0) & =0 \\
T_{1, m}(N+1) & =\tilde{b}_{m},
\end{aligned}
$$

where the matrices $\tilde{A}_{m}(\cdot)$ and the vectors $\tilde{b}_{m}$ were defined in (111) and (124), respectively.

For the sake of convenience, problem (127) with boundary condition (128), is solved by forward recurrence from $i=1$ up to $i=N$ :

$$
\begin{aligned}
\vec{T}_{m}(i) & =\tilde{A}_{m}(i) \vec{T}_{m}(i-1)=\tilde{A}_{m}(i) \tilde{A}_{m}(i-1) \vec{T}_{m}(i-2) \\
& =\tilde{A}_{m}(i) \tilde{A}_{m}(i-1) \tilde{A}_{m}(i-2) \vec{T}_{m}(i-3) \\
& =\cdots \\
& =\tilde{A}_{m}(i) \tilde{A}_{m}(i-1) \tilde{A}_{m}(i-2) \cdots \tilde{A}_{m}(i-(i-2)) \tilde{A}_{m}(i-(i-1)) \vec{T}_{m}(0) \\
& =\prod_{\ell=0}^{i-1} \tilde{A}_{m}(i-\ell)\left[\begin{array}{c}
0 \\
T_{2, m}(0)
\end{array}\right], \quad 1 \leq i \leq N+1 .
\end{aligned}
$$

For the free component $T_{2, m}(0)$ in (130), let us take the value $T_{2, m}(0)=1$ and

$$
\overrightarrow{\hat{Y}}_{m}(i)=\prod_{\ell=0}^{i-1} \tilde{A}_{m}(i-\ell)\left[\begin{array}{l}
0 \\
1
\end{array}\right], \quad 1 \leq i \leq N+1 .
$$

Note that by construction its first component $[1,0] \overrightarrow{\hat{Y}}_{m}(i)$ satisfies (128). Following the shooting method ideas, and taking into account that from (112) one gets

$$
\overrightarrow{\hat{v}}_{m}:=\overrightarrow{\hat{Y}}_{m}(0) \neq \mathbf{0}
$$


and provided that $[1,0] \vec{v}_{m} \neq 0$, then

$$
T_{1, m}(i)=\frac{\tilde{b}_{m}}{[1,0] \vec{v}_{m}}[1,0] \vec{Y}_{m}(i), \quad 0 \leq i \leq N+1,
$$

satisfies (127)-(129), for each $m, 1 \leq m \leq M$. Finally, undoing the variable change $T_{1, m}(i):=G_{m}^{\mathrm{IV}}(i)$ and by (96) we have constructed a solution for the case IV:

$$
\mathbf{U}^{\mathrm{IV}}(i, j)=\sum_{m=1}^{M} \tilde{H}_{m}^{\mathrm{III}}(j) T_{1, m}(i), \quad 1 \leq i \leq N, \quad 1 \leq j \leq M,
$$

where $\left\{T_{1, m}(\cdot)\right\}_{m=1}^{M}$ are obtained from (131)-(133).

\section{Solution for Cases III and IV.}

By the linearity of Eq. (1) and the superposition principle, the constructed closed form numerical solution for both cases III-IV is

$$
\mathbf{U}^{\mathrm{III}}(i, j)+\mathbf{U}^{\mathrm{IV}}(i, j)=\sum_{m=1}^{M} \tilde{H}_{m}^{\mathrm{III}}(j)\left(L_{1, m}(i)+T_{1, m}(i)\right), \quad 1 \leq i \leq N, \quad 1 \leq j \leq M,
$$

being the eigenfunctions $\left\{\tilde{H}_{m}^{\mathrm{III}}(\cdot)\right\}_{m=1}^{M}$ those obtained from orthonormalizing the eigenfunctions $\left\{H_{m}^{\text {III }}(\cdot)\right\}_{m=1}^{M}$ given by $(94)$, and $\left\{L_{1, m}(\cdot)\right\}_{m=1}^{M}$ and $\left\{T_{1, m}(\cdot)\right\}_{m=1}^{M}$ the expressions which are obtained from (117)-(119) and (131)-(133), respectively.

\section{Constructive numerical algorithm and validation}

By sections 3 and 4 (see (67) and (135)), the linearity of Eq. (1) and the superposition principle, we have constructed a closed form numerical solution for the elliptic problem (1)-(6):

$$
\begin{aligned}
\mathbf{U}(i, j)=\sum_{n=1}^{N} H_{n}(i)\left[c_{n}^{I}\left(z_{1, n}^{j}-z_{1, n}^{2 M+2} z_{2, n}^{j}\right)+c_{n}^{I I}\left(z_{1, n}^{j}-z_{2, n}^{j}\right)\right] & \\
& +\sum_{m=1}^{M} \tilde{H}_{m}^{\mathrm{III}}(j)\left(L_{1, m}(i)+T_{1, m}(i)\right), \quad 1 \leq i \leq N, \quad 1 \leq j \leq M,
\end{aligned}
$$

which elements were defined in sections 3 and 4.

\subsection{Constructive algorithm}

Step 1. Discretization computation.

- Choose the number of internal mesh points $N$ and $M$ for both axis $x$ and $y$, and compute their respective step-sizes $h=\frac{b-a}{N+1}$ and $k=\frac{d-c}{M+1}$.

- Generate the mesh points $x_{i}=a+i h$ and $y_{j}=c+j k$ for $1 \leq i \leq N$ and $1 \leq i \leq M$, respectively.

- Compute $\alpha=\frac{k}{h}, r(i), p(i)$ and $q(i)$ for $0 \leq i \leq N$.

Step 2. Computation of the solution for the boundary conditions of cases I-II.

- Compute the eigenpairs $\left(\lambda_{n}, H_{n}(i)\right)_{n=1}^{N}$ for $1 \leq i \leq N$ of the algebraic eigenvalue problem (39). 
- Orthonormalize the computed eigenfunctions $\left\{H_{n}(\cdot)\right\}$.

- Compute the two simple real roots $z_{1, n}$ and $z_{2, n}$, for each computed eigenvalue $\left\{\lambda_{n}\right\}_{n=1}^{N}$, using (44).

- Compute the functions involved in boundary conditions $f_{2}(i)$ and $g_{2}(i)$ for $0 \leq i \leq N+1$.

- Compute the coefficients $c_{n}^{I}$ and $c_{n}^{I I}$, for each $n, 1 \leq n \leq N$, using (56) and (66), respectively.

- Compute the solution $\mathbf{U}^{I}(i, j)+\mathbf{U}^{I I}(i, j)$ for $1 \leq i \leq N, 1 \leq j \leq M$, using (67).

Step 3. Computation of the solution for the boundary conditions of cases III-IV.

- Compute the eigenpairs $\left(\hat{\lambda}_{m}, H_{m}^{\mathrm{III}}(j)\right)_{m=1}^{M}$ for $1 \leq j \leq M$, using (94)-(95).

- Orthonormalize the computed eigenfunctions $\left\{H_{m}^{\mathrm{III}}(\cdot)\right\}$, denoting them as $\left\{\tilde{H}_{m}^{\mathrm{III}}(\cdot)\right\}$.

- Compute the functions involved in boundary conditions $f_{1}(j)$ and $g_{1}(j)$ for $0 \leq j \leq M+1$.

- Compute the coefficients $\tilde{a}_{m}$ and $\tilde{b}_{m}$, for $1 \leq m \leq M$, using (101) and (124), respectively.

- Compute the values $a_{0}(i)$ and $a_{2}(i)$ for $1 \leq i \leq N$ using (104). Compute, for each $m$, with $1 \leq m \leq M$, the values $a_{1, m}(i)$ for $1 \leq i \leq N$ using (104).

- Compute, for each $m, 1 \leq m \leq M$, the values $L_{1, m}(i)$ for $0 \leq i \leq N$ using (117)-(119).

- Compute, for each $m, 1 \leq m \leq M$, the values $T_{1, m}(i)$ for $1 \leq i \leq N+1$ using (131)-(133).

- Compute the solution $\mathbf{U}^{\mathrm{III}}(i, j)+\mathbf{U}^{\mathrm{IV}}(i, j)$ for $1 \leq i \leq N, 1 \leq j \leq M$, using (135).

Step 4. Compute the numerical solution $\mathbf{U}(i, j)$ of the elliptic problem (1)-(6) by adding the computed solutions in steps 2-3: $\mathbf{U}(i, j)=\mathbf{U}^{\mathrm{I}}(i, j)+\mathbf{U}^{\mathrm{II}}(i, j)+\mathbf{U}^{\mathrm{III}}(i, j)+\mathbf{U}^{\mathrm{IV}}(i, j)$, for $1 \leq i \leq N$ and $1 \leq j \leq M$ using (136). Compute $\mathbf{U}(i, j)$ for $i=0, i=N+1, j=0$ and $j=M+1$ using the functions involved in boundary conditions.

Taking into account [21] and the previous algorithm, it is easy to show that the computational complexity of the closed form numerical solution (136) is $\mathrm{O}\left(N^{3}+M^{2}\right)$, being $N$ and $M$ the number of internal mesh points on axis $x$ and $y$, respectively.

\subsection{Numerical example}

Consider the elliptic problem

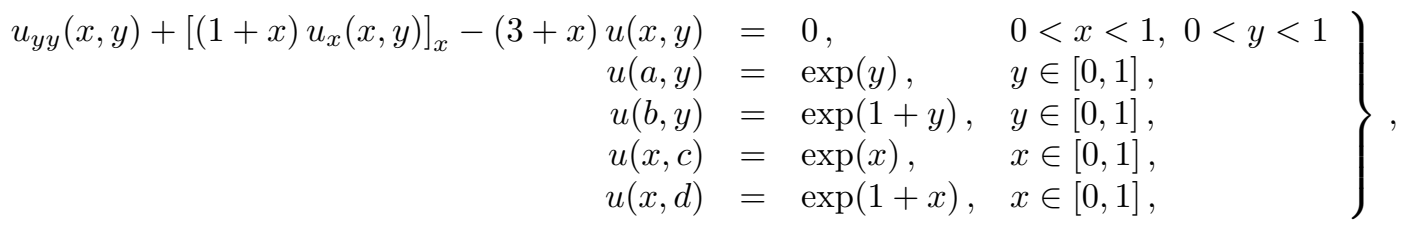

satisfying hypotheses given by (6). The exact solution of this problem is

$$
u(x, t)=\exp (x+y), \quad 0 \leq x \leq 1, \quad 0 \leq y \leq 1 .
$$

In figure 1 we can see that the numerical solution $\mathbf{U}(i, j)$ given by expression (136) reproduces very well the behavior of the exact solution $u\left(x_{i}, y_{j}\right)$ given by (138), taking only $M=3$ internal mesh points on axis $y$. For this simulation, the absolute error in each mesh point $(i, j)$ is shown in figure 2. Furthermore, as $M \ll N$ the computational complexity (see section 5.1) is $\mathrm{O}\left(N^{3}\right)$ which is related to the task of computing the eigenpairs $\left(\lambda_{n}, H_{n}(i)\right)_{n=1}^{N}$ of Sturm-Liouville problem (39) in cases I-II.

In order to quantify the order of the errors made respect to the exact solution (138), we consider the maximum absolute error over all mesh points, that is:

$$
\|E\|_{\infty}=\max _{i, j}\left(u\left(x_{i}, y_{j}\right)-\mathbf{U}(i, j)\right), \quad 1 \leq i \leq N, 1 \leq j \leq M .
$$


In table 1 we can see that the numerical results improve when the step-size $h$ decreases (fixed step-size $k$ ), that is, when the number of internal mesh points $N$ increases (fixed $M$ ), taking just a few internal mesh points $M$, such as $M=3$. This fact checks the consistency of explicit difference scheme (10). Note that due to the truncation error is of order $\mathrm{O}\left(h+k^{2}\right)$ we have refined in axis $x$. Furthermore, in order to obtain better approximations, it is only necessary to consider a few mesh points $N$ and $M$ because of the discrete Sturm-Liouville problems, which arise in cases I-II and in cases III-IV, retain the qualitative properties of the analytic (exact) solution (138).
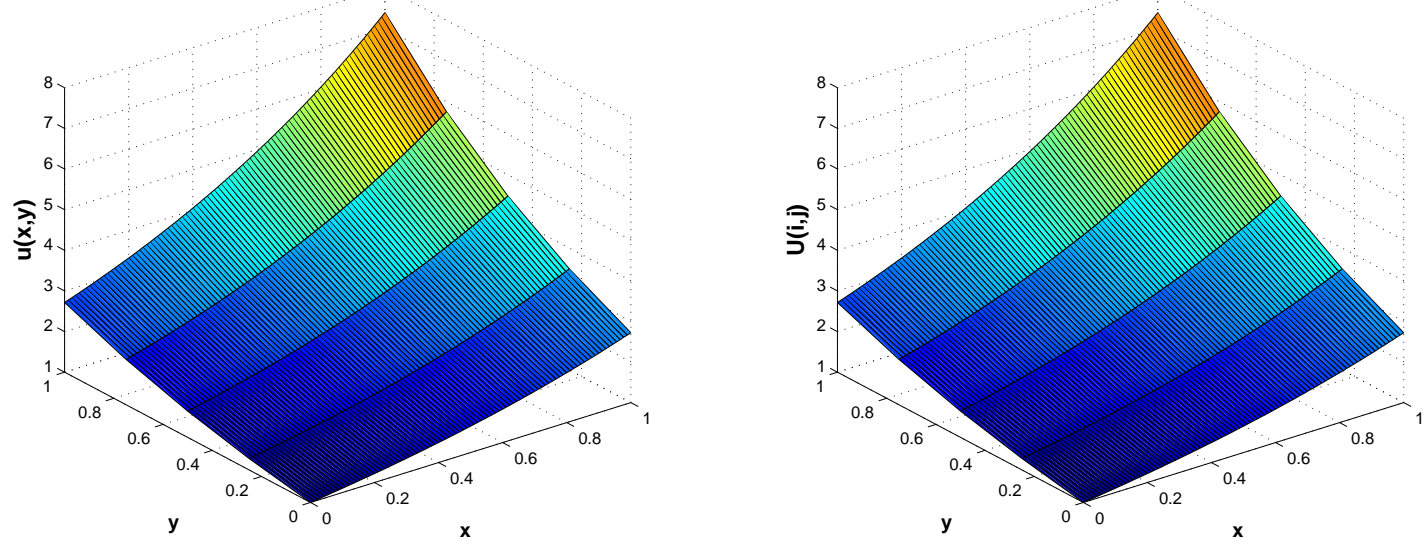

Figure 1: Comparison of the exact solution $u\left(x_{i}, y_{j}\right)$ (on the left) vs the numerical solution $\mathbf{U}(i, j)$ (on the right) obtained using (136), for the internal mesh points $N=99$ and $M=3$.

\begin{tabular}{cclcc}
\hline$N$ & $M$ & $h=\frac{b-a}{N+1}$ & $k=\frac{d-c}{M+1}$ & $\|E\|_{\infty}$ \\
\hline 3 & 3 & 0.25 & 0.25 & $1.4374 \times 10^{-2}$ \\
9 & 3 & 0.1 & 0.25 & $5.8715 \times 10^{-3}$ \\
19 & 3 & 0.05 & 0.25 & $2.6553 \times 10^{-3}$ \\
39 & 3 & 0.025 & 0.25 & $9.8568 \times 10^{-4}$ \\
99 & 3 & 0.01 & 0.25 & $3.4458 \times 10^{-5}$ \\
\hline
\end{tabular}

Table 1: The maximum absolute error for several values of $N$ and $M$.

\section{Concluding remarks}

This article provides an important innovation: the construction of a numerical solution in closed form, given by (136), for variable coefficient linear second-order elliptic problems of the type (1)-(6). A summary of the calculation process of the numerical solution (136) is detailed in section 5.1. The technique used is based on discretizing the elliptic equation (1) by an explicit finite difference and applying a discrete separation of variables method to the resulting explicit scheme which has $\mathrm{O}\left(h+k^{2}\right)$ local truncation error. Due to the accuracy of the constructed scheme $(10)$ is $\mathrm{O}(h)$ for variable $x$ and $\mathrm{O}\left(k^{2}\right)$ for variable $y$, more refinement in variable $x$ than in $y$ is required in order to obtain better approximations. The numerical results illustrate this fact. Furthermore, the underlying discrete Sturm-Liouville problems, in cases I-II and cases III-IV for the boundary conditions, preserve the qualitative properties of the analytic (exact) 


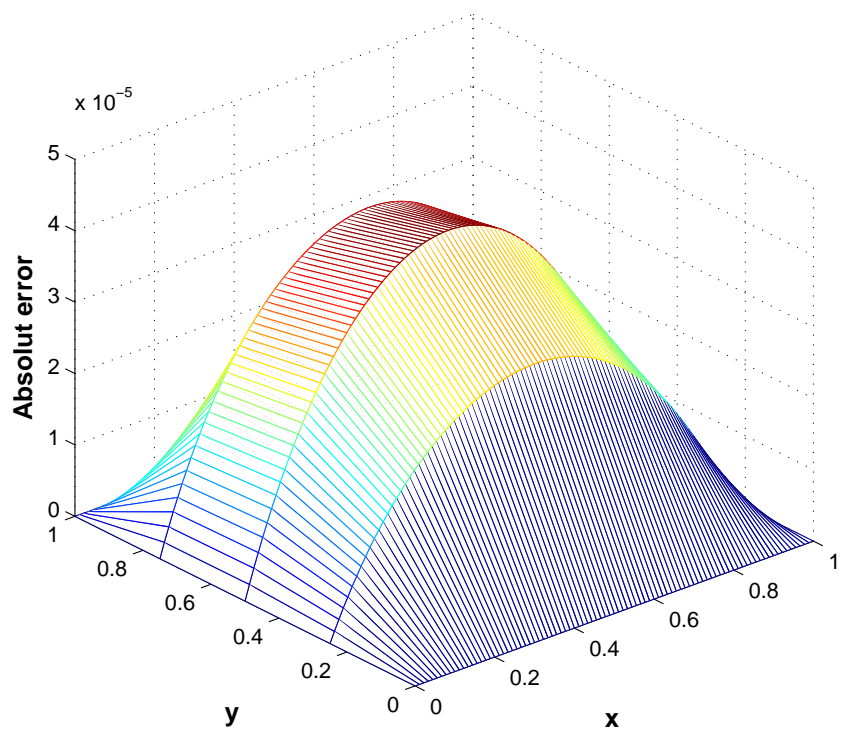

Figure 2: Graph of the absolute error: $\left|u\left(x_{i}, y_{i}\right)-U(i, j)\right|$, in each mesh point $\left(x_{i}, y_{j}\right)$ for $N=99$ and $M=3$.

solution. Thus, it is only necessary to take a few internal mesh points $N$ and $M$ for having good results, as it is shown in the numerical example. Comparing with results of [7] the local truncation error is of the same order in variable $x$ and higher accurate in variable $y$. Because of qualitative properties of the numerical solution (136) with just a few nodes the quality of the approximation is better, saving computational cost. The numerical technique developed can be extended to non-homogeneous elliptic problems.

\section{References}

[1] S.Y. Reutskiy, The method of approximate fundamental solutions (MAFS) for elliptic equations of general type with variable coefficients, Engineering Analysis with Boundary Elements. 36 (2012) 985-992.

[2] XT. Xiong, M. Li, M.Q. Wang, A one-stage meshless method for nonhomogeneous Cauchy problems of elliptic partial differential equations with variable coefficients, Journal of Engineering Mathematics 80 (1) (2013) 189-200.

[3] H. Xu, C. Zhang, Numerical solution of a transformed parabolic equation, Applied Mathematics and Computation 139 (2003) 535-554.

[4] H. Xu, C. Zhang, R. Barron, A new numerical approach to solve an elliptic equation, Applied Mathematics and Computation 171 (2005) 1-24.

[5] U. Ananthakrishnaiah, R. Manohar, J.W. Stephenson, Fourth-order finite difference methods for three-dimensional general linear elliptic problems with variable coefficients, Numerical Methods for Partial Differential Equations 3 (3) (1987) 229-240.

[6] J. Zhao, T. Zhang, R.M. Corless, Convergence of the compact finite difference method for secondorder elliptic equations, Applied Mathematics and Computation 182 (2006) 1454-1469. 
[7] I.T. Angelova, L.G. Vulkov, High-order finite difference schemes for elliptic problems with intersecting interfaces, Applied Mathematics and Computation 187 (2007) 824-843.

[8] M. Oevermann, R. Klein, A Cartesian grid finite volume method for elliptic equations with variable coefficients and embedded interfaces, Journal of Computational Physics 219 (2) (2006) 749-769.

[9] T. Lin, X. Ye, A posteriori error estimates for finite volume method based on bilinear trial functions for the elliptic equation, Journal of Computational and Applied Mathematics 254 (2013) 185-191.

[10] S. Balasundafum, P.K. Bhattacharyy, A mixed finite element method for fourth order elliptic equations with variable coefficients, Computers and Mathematics with Applications 10 (3) (1984) 245-256.

[11] D. Yang, Finite elements for elliptic problems with wild coeficients, Mathematics and Computers in Simulation 54 (2000) 383-395.

[12] V. Vemuri, W.J. Karplus, Digital Computer Treatment of Partial Differential Equations, tenth ed., Prentice-Hall Series in Computational Mathematics, Inc., Englewood Cliffs, New Jersey 07632, 1981.

[13] G.D. Smith, Numerical Solution of Partial Differential Equations: Finite Difference Methods, third. ed., Oxford University Press Inc., New York, 2004.

[14] G. Birkhoff, G.C. Rota, Ordinary Differential Equations, 4th. Ed., John Wiley \& Sons Canada, Ltd., 1989 .

[15] R. Aloy, M.C. Casabán, L.A. Caudillo-Mata, L. Jódar, Computing the variable coefficient telegraph equation using a discrete eigenfunctions method, Computers and Mathematics with Applications 54 (2007) 448-458.

[16] R. Aloy, M.C. Casabán, L. Jódar, Constructing unconditionally time stable numerical solutions for mixed parabolic problems, Computers Mathematics with Applications 53 (2007) 1773-1783.

[17] R. Aloy, M.-C. Casabán, L. Jódar, A discrete eigenfunctions method for computing mixed hyperbolic problems based on an implicit difference scheme, Applied Mathematics and Computation 215 (2009) $333-343$.

[18] J.C. Strikwerda, Finite Difference Schemes and Partial Differential Equations, 2nd. Ed., SIAM edition, USA, 2004.

[19] R.P. Agarwal, Difference Equations and Inequalities. Theory, Methods and Applications, Marcel Dekker Inc., USA, 2000.

[20] Z. Gajić, M.T.J. Qureshi, Lyapunov matrix equation in system stability and control, Mathematics in Science and Engineering (Volume 195), Academic Press Inc., New York, 1995.

[21] G. Brassard, P. Bratley, Fundamentals of Algorithmics, Prentice Hall, 1996. 